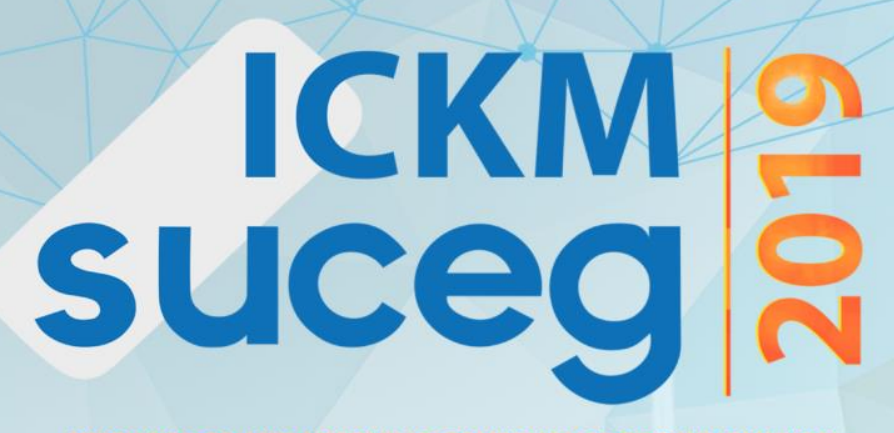

\title{
CONEXÃO ENTRE CONHECIMENTO E APRENDIZAGEM NA INDÚSTRIA 4.0: ANÁLISE DO ESTADO DA ARTE
}

\author{
DIEGO AUGUSTUS SENNA \\ Mestrando, Universidade Federal de Minas Gerias e Universidade PUC Minas, \\ https://orcid.org/0000-0002-2765-726X, augustus.senna@yahoo.com.br \\ JUREMA SUELY DE ARAÚJO NERY RIBEIRO \\ Doutora, Universidade FUMEC, Universidade UEMG e Universidade PUC Minas, \\ https://orcid.org/0000-0002-6465-6020, jurema.nery@gmail.com
}

\section{RESUMO}

Objetivo: Busca-se identificar estudos que relacionem os construtos Indústria 4.0, Aprendizado e Conhecimento e categorizá-los para analisar tendências de temas abordados, áreas de estudo, periódicos de referência e países de origem, de forma a determinar o atual estado da arte.

Design/Metodologia/Abordagem: Realizou-se pesquisa com abordagem quantitativa e inferências qualitativas, de caráter bibliográfico e bibliométrico, havendo categorização e análise estatística realizadas por meio do software Microsoft Excel e elaboração de nuvem de palavras por meio do software Wordle.

Resultados: Identificou-se que os temas de aprendizado homem-máquina e preparação acadêmica prevalecem nas publicações, que são concentradas em países com economias mais desenvolvidas.

Limitações da pesquisa: O tema é recente e o número de publicações é ainda reduzido. Além disso, o número de bases de dados pesquisadas pode ser expandido e variações linguísticas e regionais dos construtos podem ter limitado a indexação na pesquisa. 
Originalidade/valor: Estudos que relacionam a temática do conhecimento com a Indústria 4.0 são recentes, principalmente em países emergentes, e poucos realizam análises bibliométricas.

Palavras-chave: Aprendizado. Bibliometria. Conhecimento. Estado da arte. Indústria 4.0.

\title{
CONNECTION BETWEEN KNOWLEDGE AND LEARNING IN INDUSTRY 4.0: STATE OF THE ART ANALYSIS
}

\begin{abstract}
Goal: This paper seeks to identify studies that relate the Industry 4.0, Learning and Knowledge constructs and categorize them to analyze trends of topics covered, areas of study, reference journals and countries of origin, in order to determine the current state of the art.

Design / Methodology / Approach: The research was conducted with a quantitative approach and qualitative inferences of bibliographic and bibliometric character, with categorization and statistical analysis performed using Microsoft Excel software and world cloud elaboration through Wordle software.

Results: The themes of man-machine learning and academic preparation were found to prevail in publications, which are concentrated in countries with more developed economies.

Limitations of the research: The topic is recent and the number of publications is still small. In addition, the number of databases searched may be expanded and linguistic and regional variations of the terms may have limited search indexing.

Originality / value: Studies relating the theme of knowledge to Industry 4.0 are recent, especially in emerging countries, and few perform bibliometric analyzes.
\end{abstract}

Keywords: Bibliometrics. Industry 4.0. Knowledge. Learning. State of the art. 


\section{INTRODUÇÃO}

O grande dinamismo da Indústria 4.0 simboliza abrupta ruptura com modelos clássicos de negócios. As rápidas transformações alteram completamente a dinâmica de mercado e a sobrevivência das empresas depende do lançamento de novos produtos e da capacidade de adaptar estratégias (Synnes \& Welo, 2016). Os sistemas tradicionais de automação não são capazes de responder de forma adequada ao aumento exponencial da complexidade dos produtos, com simultânea redução de seu ciclo de vida (Gorecky, Khamis \& Mura, 2017).

Visando atender aos anseios de mercado, novos sistemas são propostos e com isso os funcionários precisam lidar com transformações profundas do ambiente do trabalho, por vezes sem preparação para tal. Simultaneamente, empregadores podem não fornecer condições ideais para o aprendizado de novas tarefas (Thalmann, Pammer-Schindler, Fessl \& Weghofer, 2017). O gerenciamento do conhecimento e dos mecanismos de aprendizagem apresenta-se, portanto, como critério de fundamental importância para o inevitável desenvolvimento de uma cultura digital, desafio comum a empregados e administradores (Hariharasudan \& Kot, 2018).

A Indústria 4.0 é, em sua definição, recente, mas os extensos impactos provocados já despertam profundo interesse no setor educacional, tendo em vista a rápida obsolescência do saber tradicional. Intenso treinamento em tecnologias de vanguarda é necessário para atender a requisitos desejados pelo mercado de trabalho, e métodos clássicos de ensino tendem a não ser os mais adequados (Karre, Hammer, Kleindienst \& Ramsauer, 2017). A interação do conhecimento entre seres humanos e máquinas é fator central e não pode ser dissociada ao tratar do assunto, tendo em vista a ocorrência de transição da simples cooperação para a colaboração ativa, havendo fusão de responsabilidades entre seres humanos e máquinas ao realizar tarefas (Ansari, Khobreh, Seidenberg \& Sihn, 2018).

Considerando a posição central da temática do conhecimento na Quarta Revolução Industrial, este artigo busca determinar, por meio de revisão bibliométrica, como a conexão entre o conhecimento, o aprendizado e a Indústria 4.0 tem sido realizada na literatura. Objetiva-se identificar estudos que tenham relação com o assunto e categorizá-los para analisar tendências de temas abordados, áreas de estudo e periódicos de referência. Adicionalmente, levando em consideração a existência de diferenças no nível de apropriação da Indústria 4.0 entre países, sendo o assunto mais recente para os emergentes (Silva, Kovaleski \& Pagani, 2018), procura-se determinar a distribuição de publicações conforme a nação de origem. 
Baseando-se na contemporaneidade da Indústria 4.0, no interesse acadêmico, na importância da interação entre os conhecimentos humano e de máquinas e no alcance desigual da Indústria 4.0 entre países, foram estabelecidas as seguintes hipóteses: H1) O tema do gerenciamento do conhecimento na Indústria 4.0 é recente e está sendo incorporado ao setor acadêmico; H2) A interação entre homem e máquina, que passa a ter caráter mais cooperativo na Indústria 4.0, é tema de grande interesse; H3) As publicações voltadas ao assunto estão concentradas em países do Norte.

Buscando atender aos objetivos propostos, este estudo parte do referencial teórico que sustenta os três descritores utilizados na pesquisa (item 2.1), seguido por apresentação da metodologia de identificação e classificação (item 2.2), apresentação de resultados e análises realizadas (item 2.3) e considerações finais (item 3).

\section{DESENVOLVIMENTO}

\subsection{REFERENCIAL TEÓRICO}

\subsubsection{Indústria 4.0}

Diversas das tecnologias que propiciam a revolução definida como Indústria 4.0 são consequência direta da digitalização e estão presentes no mercado há algum tempo. A “Industrie 4.0”, em definição, iniciou-se como política pública da Alemanha que estabeleceu a ligação dessas tecnologias dentro de um mesmo contexto, algo logo realizado também por outros países (Ghani \& Muhammad, 2019; Rodrigues, Jesus \& Schützer, 2016). A iniciativa americana, conhecida como Smart Manufacturing, e a sul-coreana, definida como Smart Factory Program, são exemplos de programas semelhantes, centrados no uso de dados digitais e apoiados por diversas tecnologias, como Sistemas Cyber-Físicos e Internet das Coisas (Lenz, Wuest \& Westkämper, 2018).

Essa mobilização está sendo observada em diversas das economias mais avançadas do mundo, sendo promovida pela busca incessante por aumento de produtividade e eficiência no ambiente industrial, justificada pela grande participação da manufatura como fonte geradora de renda nesses países (Baena, Guarin, Mora, Sauza \& Retat, 2017). Quanto aos desafios enfrentados, Shamim, Cang, Yu e Li (2017) apontam a necessidade de obter, em tempo hábil, informações sobre desejos dos consumidores para fornecer a combinação correta de produtos e 
serviços customizados, a consolidação de um ambiente de trabalho inteligente e o funcionamento eficiente e efetivo da cadeia de suprimentos.

Nesse contexto, Arbix, Salerno, Zancul, Amaral e Lins (2017) apontam cinco eixos de tendências, cabendo ressaltar: i) a presença de cada vez mais origens de valor adicionado a mercadorias e serviços; ii) a promoção do fluxo de conhecimento e do aprendizado; iii) a junção com agentes sociais de outros campos para a cooperação em busca do conhecimento; iv) a inclinação em poupar empregos, apesar de novos estudos serem necessários; e v) a importância da manufatura como promotora da inovação, embora com reduzida capacidade de geração de postos de trabalho.

Infere-se, portanto, que deve haver valorização cada vez maior da temática da gestão do conhecimento, tendo em vista que é a base das transformações promovidas pela Indústria 4.0 e a pressão exercida pelo mercado torna obrigatória a integração dessas novas tecnologias em processos industriais. As empresas inovadoras têm, pela frente, inúmeras oportunidades de crescimento, mas aquelas que demoram a reagir ou o fazem de maneira ineficiente enfrentam sério risco de desaparecer (Stachová, Papula, Stacho \& Kohnová, 2019).

\subsubsection{Conhecimento}

O conhecimento não se define como um simples elemento inerente à execução de processos industriais, mas sim como importante recurso que define o sucesso de um negócio. Exerce, segundo Ricalde, Ortiz, Sánchez e Bustos (2015), papel de atrativo estratégico, não escasso, mas que deve ser corretamente aplicado para que maiores oportunidades de crescimento sejam alcançadas. Romero e Araújo (2015, p. 190) apontam, para o cenário atual, a formação de um "ambiente que se caracteriza por uma economia baseada no conhecimento, em tecnologia intensiva e em constante mudança”".

Empresas campeãs de inovação estão intimamente conectadas à capacidade de desenvolver novos conhecimentos, o que depende da base pré-existente, da experiência e de habilidades individuais dos funcionários. Em diversas companhias, esse conhecimento acaba restrito a poucos empregados.

O compartilhamento surge neste contexto como forma de promover o desenvolvimento de habilidades da equipe como um todo e, para a Indústria 4.0, plataformas virtuais emergem como alternativas promissoras. Dessa forma, eleva-se o potencial de capital intelectual para ganhar competitividade no mercado, conectando não apenas os funcionários internos à companhia, mas 
também diversos atores envolvidos ao longo da cadeia de suprimentos (Kaasinen et al., 2019; Kinkel, Schemmann \& Lichtner, 2017; Stachová et al., 2019).

Segundo Diez-Olivan, Del Ser, Galar e Sierra (2018), a utilização de novas tecnologias de informação e comunicação nas indústrias manufatureiras promove a junção entre os ambientes real e virtual, havendo grande expansão da infraestrutura de Internet. Há, portanto, a construção de um conhecimento misto baseado na interação entre seres humanos e máquinas. Essa articulação híbrida é ainda campo vasto de pesquisa, e a utilização de tecnologias como realidade virtual e localização em tempo real para prover informações relevantes e conhecimento abrem novas possibilidades (Stocker, Brandl, Michalczuk \& Rosenberger, 2014; Zeidler, Bayhan, Venkatapathy \& Hompel, 2017).

\subsubsection{Aprendizado}

A temática do aprendizado na Indústria 4.0 pode ser analisada nas esferas humana, artificial ou na junção de ambas. Apesar da Quarta Revolução Industrial ser geralmente associada à produção gerenciada por computadores, o aprendizado humano atinge patamar de grande importância, sendo essencial para o desenvolvimento de fábricas inteligentes (Stocker et al., 2014).

A participação de máquinas capazes de tomar decisões por conta própria é central na manufatura inteligente, mas o trabalhador humano continua sendo o elemento de maior flexibilidade no ambiente produtivo, com necessidade de qualificação ininterrupta (Gorecky et al., 2017). Habilidades de comunicação e trabalho em equipe são frequentemente relacionadas com aquelas que devem ser alcançadas por um engenheiro (Baena et al., 2017).

Em relação à parcela artificial, o aprendizado de máquinas é um elemento indispensável à manufatura moderna. Modelos de sistemas cyber-físicos inteligentes são definidos com base na coleta e transporte de grande volume de dados entre diversos componentes eletrônicos inteligentes, que utilizam sensores e a nuvem para compreender, transmitir informações e realizar ações sem intervenção humana, sendo importante a interoperabilidade de arquiteturas de software (Morozov, Lezoche \& Panetto, 2018). Segundo Tavcar e Horváth (2019, p. 145, tradução nossa), esses sistemas, complexos e não lineares, apresentam "um conjunto de características paradigmáticas como auto-consciência, auto-adaptação, auto-evolução e auto-reprodução". Estudar o tema é, portanto, fundamental para prever que os equipamentos atuem de forma apropriada com base em seu aprendizado. 
É indiscutível que a Quarta Revolução Industrial irá exigir habilidades de trabalho diferentes e mais específicas (Barbieri, Ciabuschi, Fratocchi \& Vignoli, 2017). Boa parte dessas habilidades envolverá, inevitavelmente, a junção entre o aprendizado humano e o de máquinas.

A digitalização dos postos de trabalho demanda mudanças no setor educacional a ponto de ser necessária uma reestruturação (Chou \& Feng, 2019). Por meio dessa percepção, universidades estão buscando adequar-se, promovendo, assim, o desenvolvimento de novas abordagens e a criação de renovados ambientes de ensino (ElMaraghy, Moussa, ElMaraghy \& Abbas, 2017; Siddoo, Sawattawee, Janchai \& Thinnukool, 2019). Dentre as iniciativas, a criação de fábricas de aprendizado tem recebido grande destaque, pois essas instituições permitem a aproximação entre o funcionário e tecnologias de vanguarda que irão acompanhá-lo durante seu trabalho na indústria (Küsters, Praß \& Gloy, 2017; Uhlemann, Schock, Lehmann, Freiberger, Steinhilper, 2017; Reuter et al., 2017).

\subsection{METODOLOGIA}

O presente estudo apresenta caráter exploratório (Prodanov \& Freitas, 2013) e bibliométrico, vez que busca identificar e delinear tendências na abordagem do conhecimento e do aprendizado no contexto da Indústria 4.0, quantificando dados para demonstrar a relevância do tema (Rodrigues, Tavar, Nogueira \& Librelotto, 2016). Realizou-se, nos meses de junho e julho de 2019, pesquisa definida conforme os critérios apresentados no Quadro 1.

Quadro 1 - Critérios de pesquisa.

\begin{tabular}{|c|l|}
\hline Critério & \multicolumn{1}{|c|}{ Descrição } \\
\hline Contexto & $\begin{array}{l}\text { Gerenciamento e atualização do conhecimento e aprendizagem, nos meios } \\
\text { industrial e acadêmico, considerando o contexto da Indústria 4.0 e a interface } \\
\text { homem-máquina. }\end{array}$ \\
\hline Justificativa & $\begin{array}{l}\text { Diante do dinamismo inerente à Indústria 4.0, a sobrevivência de empresas e } \\
\text { universidades depende de sua capacidade de atualização de conhecimentos, sendo } \\
\text { o aprendizado de máquina parte integrante do saber. Através da análise de } \\
\text { publicações, objetiva-se definir o estado da arte do gerenciamento de } \\
\text { conhecimento e meios de aprendizagem, tendo em vista a rápida evolução } \\
\text { tecnológica que vem sendo propiciada pela 4a Revolução Industrial. }\end{array}$ \\
\hline $\begin{array}{c}\text { Descritores } \\
\text { pesquisados }\end{array}$ & $\begin{array}{l}\text { Considerando o título, palavras-chave ou resumo, a busca foi restrita às seguintes } \\
\text { expressões, utilizando o operador "AND": "Industry 4.0" AND "Learning" AND } \\
\text { "Knowledge". }\end{array}$ \\
\hline Categoria & Apenas artigo cientifico (article). \\
\hline Idiomas & Sem restrição (universal). \\
\hline $\begin{array}{c}\text { Fontes de } \\
\text { pesquisa }\end{array}$ & Ebsco, Scielo, Scopus, Spell. \\
\hline
\end{tabular}

Fonte: Autores (2019). 
As buscas realizadas nas plataformas Ebsco e Spell não retornaram resultados. A base Scopus apresentou 39 resultados, dos quais um foi descartado por não apresentar aderência ao tema proposto. O sistema Scielo indicou apenas um artigo, descartado por duplicidade, vez que havia sido encontrado também na plataforma Scopus.

A plataforma Scopus apresentou-se como o banco de dados mais completo, sendo a fonte dos artigos analisados na pesquisa. Os 38 estudos encontrados representam número suficiente para a busca de tendências por bibliometria. A análise foi realizada em três etapas.

Primeiramente, efetuou-se a categorização dos artigos, com elaboração de planilha no programa Microsoft Excel, destacando os aspectos básicos - título, autores, palavras-chave, ano de publicação, países relacionados à filiação universitária dos autores, periódico - e associados ao conteúdo, considerando a abordagem (qualitativa, quantitativa ou mista), a área estudada, o país abordado no estudo e a metodologia utilizada. Para cada artigo, foram extraídas as principais temáticas contextualizadas, sendo atribuída uma categoria, conforme apresentado no Quadro 2.

Quadro 2 - Categorias consideradas na análise.

\begin{tabular}{|c|l|}
\hline Categoria & \multicolumn{1}{|c|}{ Descrição } \\
\hline Aprendizado Humano & $\begin{array}{l}\text { Artigos com foco no gerenciamento de conhecimento e } \\
\text { modelos de aprendizagem para os recursos humanos no } \\
\text { ambiente de trabalho. }\end{array}$ \\
\hline Aprendizado de Máquina & $\begin{array}{l}\text { Estudos voltados ao aprendizado de máquina, abrangendo } \\
\text { tecnologias e sistemas relacionados à automatização e ao } \\
\text { conhecimento não humano no ambiente de trabalho. }\end{array}$ \\
\hline Aprendizado Homem-Máquina & $\begin{array}{l}\text { Pesquisas que discutem tanto o conhecimento humano } \\
\text { quanto o de máquina, especificando relações entre ambos no } \\
\text { ambiente de trabalho. }\end{array}$ \\
\hline Preparação Acadêmica & $\begin{array}{l}\text { Artigos voltados ao sistema educacional ou ambiente de } \\
\text { pesquisa, principalmente universitário, especificando } \\
\text { inovações relacionadas ao contexto da Indústria 4.0. }\end{array}$ \\
\hline
\end{tabular}

Fonte: Autores (2019).

Na segunda etapa, os dados foram utilizados para a construção de análises quantitativas, que indicaram relações para subsidiar inferências qualitativas. Na última etapa, uma nuvem de palavras foi criada a partir das palavras-chave dos artigos, desconsiderando diferenças entre letras maiúsculas e minúsculas, por meio do aplicativo Wordle.

\subsection{APRESENTAÇÃO E DISCUSSÃO DOS RESULTADOS}

A relação dos artigos analisados, contendo suas informações básicas para identificação, é apresentada no Quadro 3. 
Quadro 3 - Identificação dos trabalhos analisados

\begin{tabular}{|c|c|c|c|}
\hline № & Ano & Autor & Título \\
\hline 1 & 2014 & $\begin{array}{l}\text { Stocker, A., Brandl, P., Michalczuk, } \\
\text { R., Rosenberger, M. }\end{array}$ & Human-centred ICT tools for smart factories \\
\hline 2 & 2016 & Synnes, E.L., Welo, T. & $\begin{array}{l}\text { Bridging the Gap between High and Low-volume } \\
\text { Production through Enhancement of Integrative } \\
\text { Capabilities }\end{array}$ \\
\hline 3 & 2017 & $\begin{array}{l}\text { Kinkel, S., Schemmann, B., } \\
\text { Lichtner, R. }\end{array}$ & $\begin{array}{l}\text { Critical Competencies for the Innovativeness of } \\
\text { Value Creation Champions: Identifying Challenges } \\
\text { and Work-integrated Solutions }\end{array}$ \\
\hline 4 & 2017 & Shamim, S., Cang, S., Yu, H., Li, Y. & $\begin{array}{l}\text { Examining the feasibilities of Industry } 4.0 \text { for the } \\
\text { hospitality sector with the lens of management } \\
\text { practice }\end{array}$ \\
\hline 5 & 2017 & $\begin{array}{l}\text { ElMaraghy, H., Moussa, M., } \\
\text { ElMaraghy, W., Abbas, M. }\end{array}$ & $\begin{array}{l}\text { Integrated Product / System Design and Planning for } \\
\text { New Product Family in a Changeable Learning } \\
\text { Factory }\end{array}$ \\
\hline 6 & 2017 & Gorecky, D., Khamis, M., Mura, K. & $\begin{array}{l}\text { Introduction and establishment of virtual training in } \\
\text { the factory of the future }\end{array}$ \\
\hline 7 & 2017 & $\begin{array}{l}\text { Thalmann, S., Pammer-Schindler, } \\
\text { V., Fessl, A., Weghofer, F. }\end{array}$ & $\begin{array}{l}\text { Learning 4.0: Addressing challenges for employees } \\
\text { successfully }\end{array}$ \\
\hline 8 & 2017 & $\begin{array}{l}\text { Reuter, M., Oberc, H., Wannöffel, } \\
\text { M., Kreimeier, D., Klippert, J., } \\
\text { Pawlicki, P., Kuhlenkötter, B. } \\
\end{array}$ & $\begin{array}{l}\text { Learning Factories' Trainings as an Enabler of } \\
\text { Proactive Workers' Participation Regarding Industry } \\
4.0\end{array}$ \\
\hline 9 & 2017 & $\begin{array}{l}\text { Baena, F., Guarin, A., Mora, J., } \\
\text { Sauza, J., Retat, S. }\end{array}$ & Learning Factory: The Path to Industry 4.0 \\
\hline 10 & 2017 & $\begin{array}{l}\text { Hold, P., Erol, S., Reisinger, G., } \\
\text { Sihn, W. }\end{array}$ & $\begin{array}{l}\text { Planning and Evaluation of Digital Assistance } \\
\text { Systems }\end{array}$ \\
\hline 11 & 2017 & $\begin{array}{l}\text { Vila, C., Ugarte, D., Ríos, J., Abellán, } \\
\text { J.V. }\end{array}$ & $\begin{array}{l}\text { Project-based collaborative engineering learning to } \\
\text { develop Industry } 4.0 \text { skills within a PLM framework }\end{array}$ \\
\hline 12 & 2017 & $\begin{array}{l}\text { Zeidler, F., Bayhan, H., } \\
\text { Venkatapathy, A.K.R., Hompel, } \\
\text { M.T. }\end{array}$ & $\begin{array}{l}\text { Reference field for research and development of } \\
\text { novel hybrid forms of human machine interaction in } \\
\text { logistics }\end{array}$ \\
\hline 13 & 2017 & Kamp, B., Ochoa, A., Diaz, J. & $\begin{array}{l}\text { Smart servitization within the context of industrial } \\
\text { user-supplier relationships: contingencies according } \\
\text { to a machine tool manufacturer }\end{array}$ \\
\hline 14 & 2017 & Küsters, D., Praß, N., Gloy, Y.-S. & $\begin{array}{l}\text { Textile Learning Factory } 4.0 \text { - Preparing Germany's } \\
\text { Textile Industry for the Digital Future }\end{array}$ \\
\hline 15 & 2017 & Madsen, O., Møller, C. & $\begin{array}{l}\text { The AAU Smart Production Laboratory for Teaching } \\
\text { and Research in Emerging Digital Manufacturing } \\
\text { Technologies }\end{array}$ \\
\hline 16 & 2017 & $\begin{array}{l}\text { Uhlemann, T.H.-J., Schock, C., } \\
\text { Lehmann, C., Freiberger, S., } \\
\text { Steinhilper, R. }\end{array}$ & $\begin{array}{l}\text { The Digital Twin: Demonstrating the Potential of } \\
\text { Real Time Data Acquisition in Production Systems }\end{array}$ \\
\hline 17 & 2017 & $\begin{array}{l}\text { Yakovle, V.V., Khasanov, M.M., } \\
\text { Sitnikov, A.N., Prokofiev, D.O., } \\
\text { Pustovskikh, A.A., Margarit, A.S., } \\
\text { Simonov, M.V., Perets, D.S. }\end{array}$ & $\begin{array}{l}\text { The direction of cognitive technologies development } \\
\text { in the Upstream Division of Gazprom Neft Company }\end{array}$ \\
\hline
\end{tabular}




\begin{tabular}{|c|c|c|c|}
\hline 18 & 2017 & $\begin{array}{l}\text { Karre, H., Hammer, M., Kleindienst, } \\
\text { M., Ramsauer, C. }\end{array}$ & $\begin{array}{l}\text { Transition towards an Industry } 4.0 \text { State of the } \\
\text { LeanLab at Graz University of Technology }\end{array}$ \\
\hline 19 & 2018 & $\begin{array}{l}\text { Ansari, F., Khobreh, M., } \\
\text { Seidenberg, U., Sihn, W. }\end{array}$ & $\begin{array}{l}\text { A problem-solving ontology for human-centered } \\
\text { cyber physical production systems }\end{array}$ \\
\hline 20 & 2018 & Hariharasudan, A., Kot, S. & $\begin{array}{l}\text { A Scoping Review on Digital English and Education } \\
4.0 \text { for Industry } 4.0\end{array}$ \\
\hline 21 & 2018 & Lenz, J., Wuest, T., Westkämper, E. & Holistic approach to machine tool data analytics \\
\hline 22 & 2018 & $\begin{array}{l}\text { Chong, S., Pan, G.-T., Chin, J., } \\
\text { Show, P.L., Yang, T.C.K., Huang, C.- } \\
\text { M. }\end{array}$ & $\begin{array}{l}\text { Integration of 3D Printing and Industry } 4.0 \text { into } \\
\text { Engineering Teaching }\end{array}$ \\
\hline 23 & 2018 & $\begin{array}{l}\text { Morozov, D., Lezoche, M., Panetto, } \\
\text { H. }\end{array}$ & Multi-paradigm modelling of Cyber-Physical Systems \\
\hline 24 & 2018 & Gitelman, L.D., Kozhevnikov, M.V. & $\begin{array}{l}\text { Paradigm of Managerial Education for a } \\
\text { Technological Breakthrough in the Economy }\end{array}$ \\
\hline 25 & 2018 & de Sá Carvalho, E.S., Filho, N.F.D. & $\begin{array}{l}\text { Proposal for a mobile learning system focusing on } \\
\text { the characteristics and applications practical of } \\
\text { industry } 4.0\end{array}$ \\
\hline 26 & 2018 & $\begin{array}{l}\text { Guo, Q., Miyamae, Y., Wang, Z., } \\
\text { Taniuchi, K., Yang, H., Liu, Y. }\end{array}$ & $\begin{array}{l}\text { Senvis-Net: Learning from imbalanced machinery } \\
\text { data by transferring visual element detectors }\end{array}$ \\
\hline 27 & 2018 & $\begin{array}{l}\text { Barbieri, P., Ciabuschi, F., } \\
\text { Fratocchi, L., Vignoli, M. }\end{array}$ & What do we know about manufacturing reshoring? \\
\hline 28 & 2019 & Tavcar, J., Horvath, I. & $\begin{array}{l}\text { A review of the principles of designing smart cyber- } \\
\text { physical systems for run-time adaptation: Learned } \\
\text { lessons and open issues }\end{array}$ \\
\hline 29 & 2019 & $\begin{array}{l}\text { Siddoo, V., Sawattawee, J., Janchai, } \\
\text { W., Thinnukool, O. }\end{array}$ & $\begin{array}{l}\text { An exploratory study of digital workforce } \\
\text { competency in Thailand }\end{array}$ \\
\hline 30 & 2019 & Lu, Y., Xie, R., Liang, S.Y. & $\begin{array}{l}\text { Bearing fault diagnosis with nonlinear adaptive } \\
\text { dictionary learning }\end{array}$ \\
\hline 31 & 2019 & $\begin{array}{l}\text { Diez-Olivan, A., Del Ser, J., Galar, } \\
\text { D., Sierra, B. }\end{array}$ & $\begin{array}{l}\text { Data fusion and machine learning for industrial } \\
\text { prognosis: Trends and perspectives towards Industry } \\
4.0\end{array}$ \\
\hline 32 & 2019 & $\begin{array}{l}\text { Longo, F., Nicoletti, L., Padovano, } \\
\text { A. }\end{array}$ & $\begin{array}{l}\text { Emergency preparedness in industrial plants: A } \\
\text { forward-looking solution based on industry } 4.0 \\
\text { enabling technologies }\end{array}$ \\
\hline 33 & 2019 & $\begin{array}{l}\text { Kaasinen, E., Schmalfuß, F., Özturk, } \\
\text { C., Aromaa, S., Boubekeur, M., } \\
\text { Heilala, J., Heikkilä, P., Kuula, T., } \\
\text { Liinasuo, M., Mach, S., Mehta, R., } \\
\text { Petäjä, E., Walter, T. }\end{array}$ & $\begin{array}{l}\text { Empowering and engaging industrial workers with } \\
\text { Operator } 4.0 \text { solutions }\end{array}$ \\
\hline 34 & 2019 & $\begin{array}{l}\text { Stachová, K., Papula, J., Stacho, Z., } \\
\text { Kohnová, L. }\end{array}$ & $\begin{array}{l}\text { External partnerships in employee education and } \\
\text { development as the key to facing industry } 4.0 \\
\text { challenges }\end{array}$ \\
\hline 35 & 2019 & Szalavetz, A. & $\begin{array}{l}\text { Industry } 4.0 \text { and capability development in } \\
\text { manufacturing subsidiaries }\end{array}$ \\
\hline 36 & 2019 & Ghani, E.K., Muhammad, K. & $\begin{array}{l}\text { Industry 4.0: Employers' expectations of accounting } \\
\text { graduates and its implications on teaching and } \\
\text { learning practices }\end{array}$ \\
\hline
\end{tabular}




\begin{tabular}{|l|l|l|l|}
\hline 37 & 2019 & Ansari, F., Glawar, R., Nemeth, T. & $\begin{array}{l}\text { PriMa: a prescriptive maintenance model for cyber- } \\
\text { physical production systems }\end{array}$ \\
\hline 38 & 2019 & Chou, P.-N., Feng, S.-T. & $\begin{array}{l}\text { Using a tablet computer application to advance high } \\
\text { school students' laboratory learning experiences: A } \\
\text { focus on electrical engineering education }\end{array}$ \\
\hline
\end{tabular}

Fonte: Autores (2019).

A análise do Quadro 3 indica que a composição autoral do material é totalmente variável, sem a presença de pesquisadores dedicados ao tema, o que pode indicar aderência à primeira hipótese do estudo, tendo em vista que a formação de redes de pesquisa em torno de assuntos recentes pode demorar algum tempo para ser consolidada. Apenas os autores Ansari, F. (artigos 19 e 37) e Sihn, W. (artigos 10 e 19) participaram de duas publicações. A Figura 1 apresenta a distribuição dos artigos por categorias ao longo do tempo.

Figura 1 - Número de publicações por ano, em função da categoria.

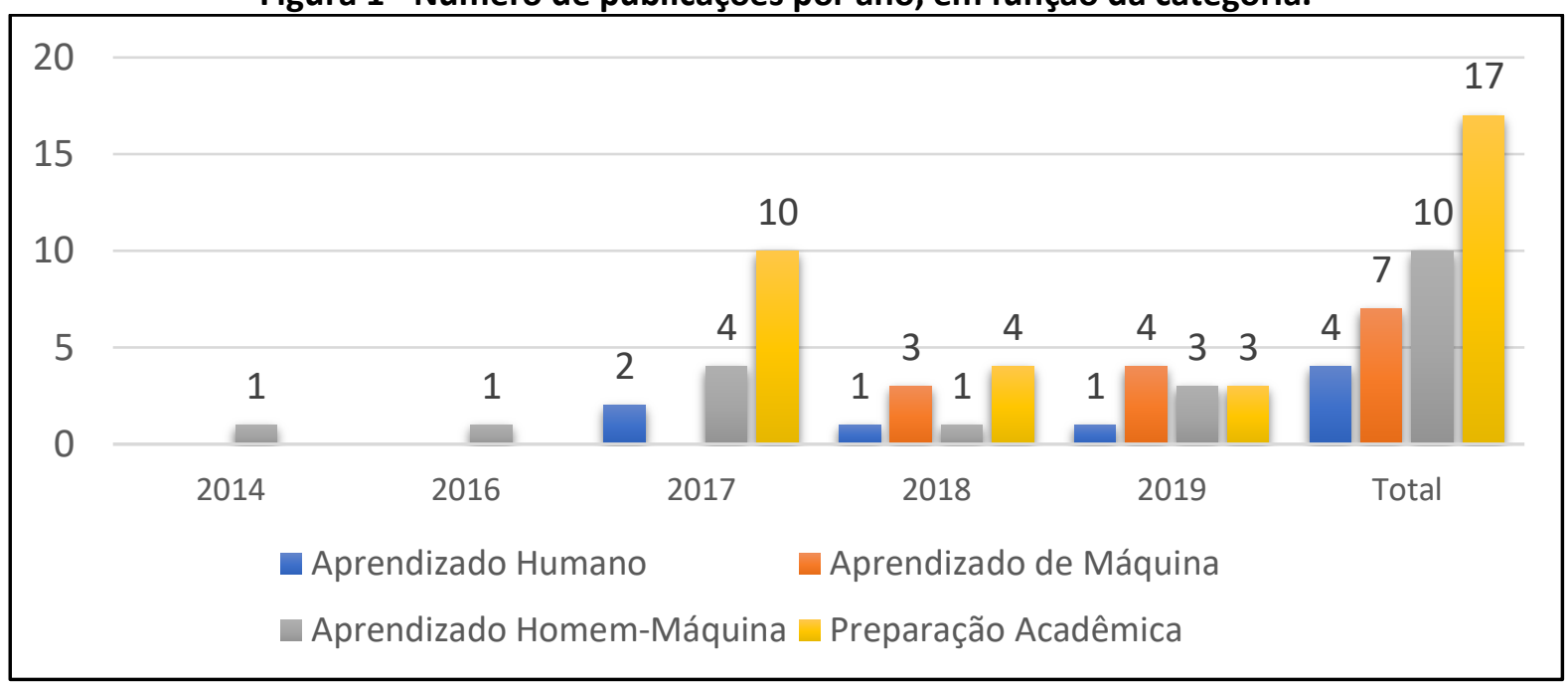

Fonte: Autores (2019).

De acordo com a Figura 1, considerando os 38 artigos analisados, 36 (94,7\%) foram publicados há menos de três anos, o que confirma a contemporaneidade do tema, sendo o ano de 2019 contabilizado apenas para os sete primeiros meses. Com relação à categorização, 21 trabalhos (55,3\%) são direcionados aos setores industrial e de negócios, sendo 10 (26,3\%) relacionados à interface do aprendizado entre trabalhadores humanos e robôs, 7 (18,4\%) direcionados ao aprendizado de máquinas e $4(10,6 \%)$ relativos ao conhecimento humano. Os 17 documentos restantes $(44,7 \%)$ apresentam foco no setor educacional, o que confirma a primeira hipótese. 
A predominância de trabalhos que abordam a interface do Aprendizado Homem-Máquina em relação aos que analisam os assuntos separadamente indica concordância com a segunda hipótese, havendo conexão mais frequente dos temas conforme ocorre a expansão da Indústria 4.0. Uma das características mais marcantes da Quarta Revolução Industrial é a aparente fusão entre os mundos real e virtual, tornando-os indissociáveis e afetando as relações de trabalho.

A abordagem utilizada por 24 artigos $(63,2 \%)$ é qualitativa, sendo a aproximação mista utilizada em 11 trabalhos $(28,9 \%)$ e a análise quantitativa aplicada em outros três (7,9\%), conforme apresentado na Figura 2a. Predominam, portanto, técnicas qualitativas de pesquisa.

Figura 2 - Distribuição de publicações: a) Por abordagem; b) Por área abordada.

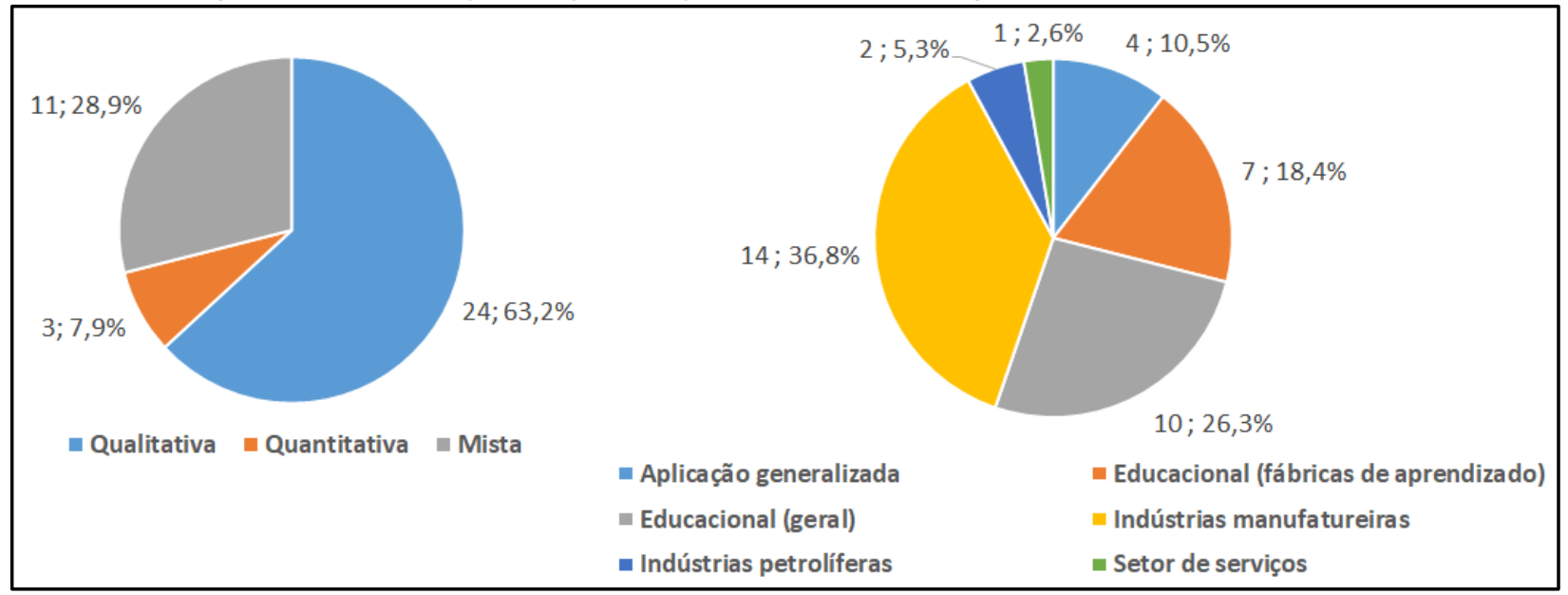

Fonte: Autores (2019).

Com relação à área abordada, conforme a Figura 2b, o setor educacional é predominante, com 17 trabalhos (44,7\%), divididos entre 7 artigos $(18,4 \%)$ que mencionam fábricas de aprendizado e $10(26,3 \%)$ que se referem às escolas, universidades e centros de pesquisa de forma generalizada. Pode-se inferir que há tendência de crescimento no desenvolvimento de estudos sobre a capacitação para a Indústria 4.0.

A Figura $2 \mathrm{~b}$ indica que as indústrias manufatureiras foram abordadas por 14 artigos $(36,8 \%)$ e indústrias petrolíferas foram o foco de outros dois (5,3\%). Apenas um documento $(2,6 \%)$ trouxe discussão voltada ao setor de serviços, o que demonstra que a área é pouco estudada na abordagem do conhecimento para a Indústria 4.0, conforme apontado por Shamim et al. (2017). Por fim, quatro trabalhos (10,5\%) utilizaram abordagem mais generalizada, sem adesão direta à uma área específica.

Apenas três periódicos estão associados a mais de um artigo, com destaque para o Procedia Manufacturing, responsável por 11 publicações (28,9\%). O Sustainability (Switzerland) 
está associado a três trabalhos (7,9\%), enquanto o International Journal of Computer Integrated Manufacturing está associado a dois (5,3\%). Nenhuma publicação foi realizada em periódico brasileiro. A relação de publicações é apresentada na Tabela 1.

Tabela 1 - Relação de publicações por periódico.

\begin{tabular}{lcc}
\hline \multicolumn{1}{c}{ Periódico } & $\begin{array}{c}\text { Número de } \\
\text { publicações }\end{array}$ & $\begin{array}{c}\text { Porcentagem } \\
\text { acumulada }\end{array}$ \\
\hline Procedia Manufacturing. & $11(28,9 \%)$ & $28,9 \%$ \\
\hline Sustainability (Switzerland). & $3(7,9 \%)$ & $36,8 \%$ \\
\hline International Journal of Computer Integrated Manufacturing. & $2(5,3 \%)$ & $42,1 \%$ \\
\hline CIRP Journal of Manufacturing Science and Technology; Computers and & \\
Industrial Engineering; Computers in Industry; Economy of Region; \\
Elektrotechnik und Informationstechnik; Energies; Heliyon; IEEE \\
Transactions on Systems, Man, and Cybernetics: Systems; IFAC- \\
PapersOnLine; Information Fusion; International Journal of Advanced \\
Manufacturing Technology; International Journal of Education and \\
Practice; International Journal of Machine Learning and Computing; \\
International Journal on Interactive Design and Manufacturing; Journal of \\
Global Operations and Strategic Sourcing; Journal of Manufacturing \\
Systems; Logistics Journal; Neftyanoe Khozyaystvo - Oil Industry; \\
Productivity Management; RISTI - Revista Ibérica de Sistemas e \\
Tecnologias de Informação; Social Sciences; Technological Forecasting and \\
Social Change.
\end{tabular}

$$
\text { Fonte: Autores (2019). }
$$

Com relação à origem, apenas seis países apresentam mais de uma publicação, conforme demonstrado na Figura 3a. Há destaque para a Alemanha, com sete publicações $(18,4 \%)$ e a Áustria, com seis publicações $(15,8 \%)$. O desenvolvimento de estudos relacionados à Indústria 4.0 - incluindo a criação do próprio termo - teve início na Alemanha, sendo esperado que o País alcance posição de evidência, assim como outras nações europeias.

Com relação aos Estados Unidos e a China, seria esperado grande número de publicações, mas há apenas um documento relacionado a cada país. Esse resultado pode ser justificado pelo uso de termos distintos. Arbix et al. (2017) apontam que a Indústria 4.0, como é conhecida na Alemanha, é tradada pelo termo Advanced Manufacturing nos Estados Unidos, enquanto DiezOlivan et al. (2018) indicam o uso do termo Industrial Internet. A variabilidade de termos dificulta a identificação e quantificação de estudos realizados nesses países, tendo em vista o caráter disperso das pesquisas. 
Figura 3 - Distribuição de publicações: a) Por países; b) Por aglomeração de países.

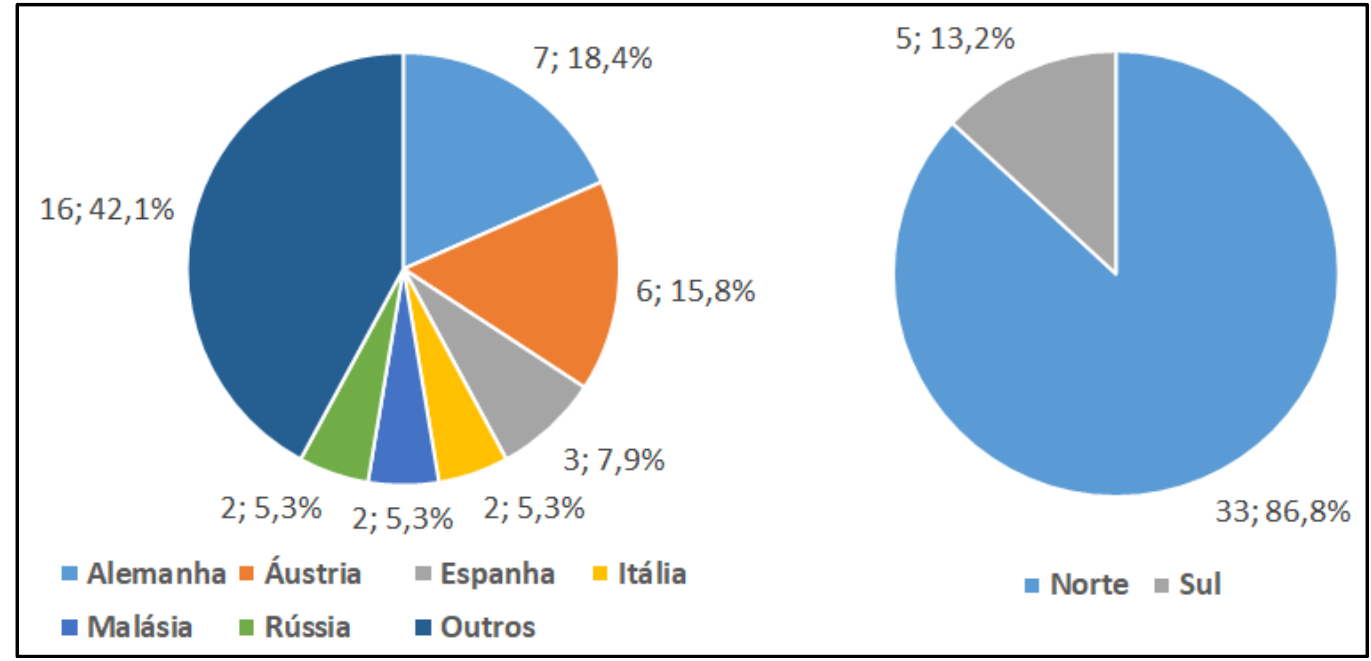

Fonte: Autores (2019).

Cabe destacar, também, que 33 artigos (86,8\%) estão associados a países do Norte, demonstrando o distanciamento com relação a países do Sul, que apresentam atraso no desenvolvimento de pesquisas relacionadas ao tema. Confirma-se, portanto, a terceira hipótese. Os resultados são apresentados na Figura 3b.

Por fim, a nuvem de palavras construída a partir das palavras-chave dos artigos está apresentada na Figura 4.

Figura 4 - Nuvem de palavras criada a partir das palavras-chave

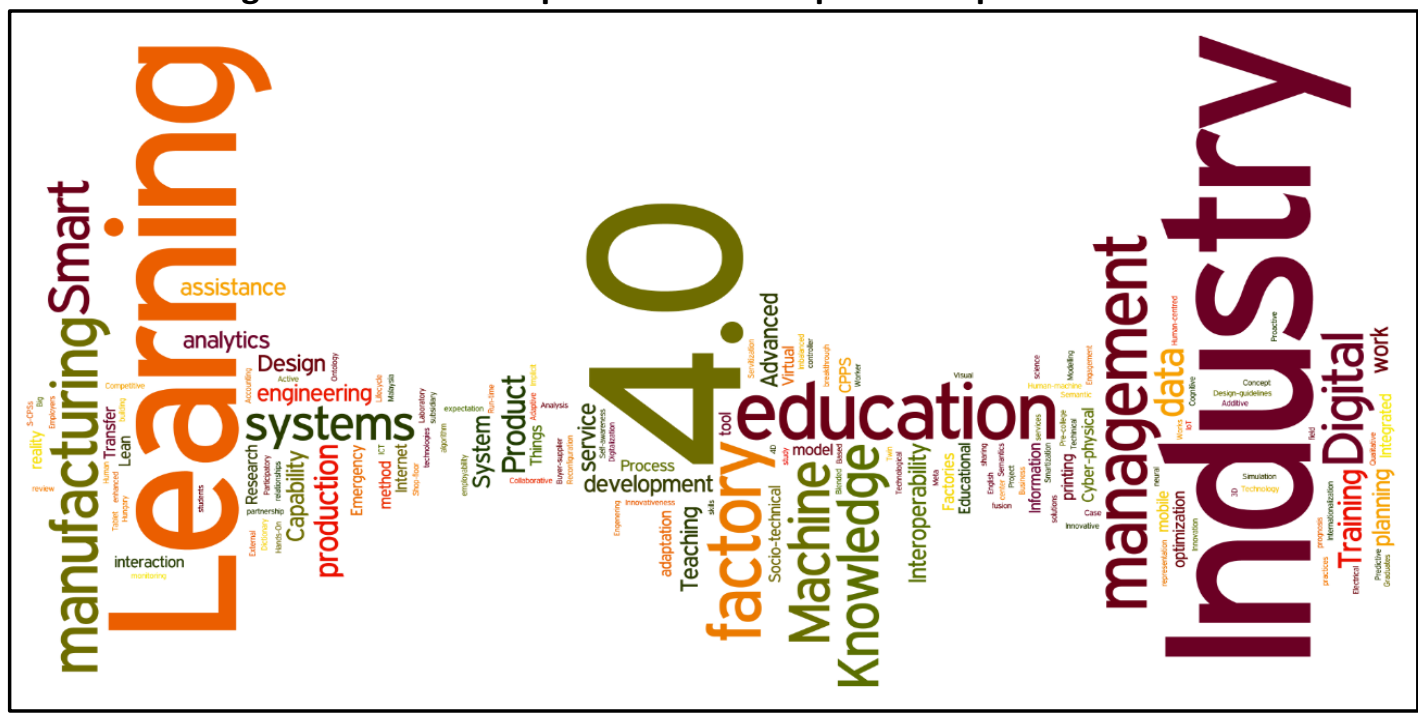

Fonte: Autores (2019).

A forte presença do setor educacional é ressaltada por expressões como education, teaching e engineering, enquanto o setor industrial manufatureiro é representado principalmente 
pelas palavras factory e manufacturing. A tendência de pesquisa voltada ao setor tecnológico é evidenciada majoritariamente pelos termos digital, machine, smart, systems, data e interoperability. A importância da temática da gestão do conhecimento nas indústrias pode ter relação com expressões como management e training. Cabe mencionar, ainda, a presença de termos voltados à gestão da produção, como product, production, capability e planning.

\section{CONSIDERAÇÕES FINAIS}

A análise dos resultados indica que, embora exista interesse das indústrias e da academia em pesquisar questões relativas ao conhecimento e ao aprendizado na Indústria 4.0, o número de publicações é ainda consideravelmente pequeno. Há, portanto, amplo espaço para o desenvolvimento de trabalhos que associem os temas. Comprovou-se que o assunto é recente e que a formação de grupos de pesquisa dedicados precisa ser consolidada.

Destacam-se, nas pesquisas, assuntos relacionados à ligação entre humanos e máquinas como forma de aprendizado e colaboração, havendo a disseminação e a junção entre o conhecimento orgânico e o artificial. Nesse contexto, destacou-se, na academia, a utilização específica de fábricas de aprendizado, que surgem como metodologia muito valorizada para promover o contato entre estudantes e as tecnologias mais recentes. Cabe mencionar, ainda, a existência de apenas uma publicação relacionada ao setor de serviços, o que demonstra afastamento em relação ao tema, apesar dos impactos relacionados à Indústria 4.0 serem refletidos nas atividades econômicas como um todo: os Serviços 4.0 dependem de modelos cada vez mais inovadores e centrados no consumidor (Nagy, Oláh, Erdei, Máte \& Popp, 2018).

Ao avaliar a origem dos trabalhos, percebe-se que há notável distanciamento entre o número de publicações associadas às economias mais desenvolvidas e aquele relacionado aos países em desenvolvimento. O fato tem ligação com o menor desenvolvimento industrial típico desses países, o que também representa reduzido potencial econômico associado ao setor. Além de haver insuficiente proatividade das indústrias na criação de pesquisas, o setor acadêmico acaba por adiar a implementação profunda da temática aos cursos, tendo em vista que existe menor pressão de mercado para a formação de novos profissionais.

O distanciamento entre países do Norte e países do Sul está associado à Indústria 4.0 como um todo e se apresenta como barreira a ser vencida. A redução das diferenças depende da criação de iniciativas que promovam a disseminação do conhecimento sobre a Quarta Revolução 
Industrial, a serem implantadas rapidamente. No mundo moderno, informação é poder e, portanto, os países que detiverem maior conhecimento sobre o assunto serão capazes de consolidar uma cultura digital e irão ocupar posições de destaque no cenário econômico internacional. A automação tradicional mostra-se cada vez mais distante de cumprir com os desejos de mercado e a atualização para a Indústria 4.0 é inevitável.

Cabe mencionar, como limitações da pesquisa, o número de artigos analisados - que pode ser ampliado no futuro, conforme novos estudos forem publicados, melhorando a representatividade da amostra - e a busca realizada em algumas bases de dados, que pode ser expandida considerando outras fontes. Variações dos termos utilizados como descritores podem também impactar no número de artigos encontrados, considerando sinônimos e variações linguísticas e regionais.

Conclui-se que os objetivos do artigo foram atingidos, vez que foram categorizados e contemplados nas análises os temas, as áreas de estudo, os periódicos e os países de origem das publicações identificadas. As hipóteses $\mathrm{H} 1$ e H2 foram confirmadas através da análise conjunta das Figuras 1 e 2, enquanto a hipótese H3 foi verificada na Figura 3.

\section{REFERÊNCIAS}

Ansari, F., Glawar, R., \& Nemeth, T. (2019). PriMa: a prescriptive maintenance model for cyberphysical production systems. International Journal of Computer Integrated Manufacturing, 32(45), 482-503.

Ansari, F., Khobreh, M., Seidenberg, U., \& Sihn, W. (2018). A problem-solving ontology for human-centered cyber physical production systems. CIRP Journal of Manufacturing Science and Technology, 22, 91-106.

Arbix, G., Salerno, M. S., Zancul, E., Amaral, G., \&Lins, L. M. (2017). Advanced Manufacturing: What Is to Be Learnt from Germany, the U.S., and China [O Brasil e a nova onda de manufatura avançada]. Novos Estudos, 36(3), 29-49.

Baena, F., Guarin, A., Mora, J., Sauza, \& J., Retat, S. (2017). Learning Factory: The Path to Industry 4.0. Procedia Manufacturing, 9, 73-80.

Barbieri, P., Ciabuschi, F., Fratocchi, L., \&Vignoli, M. (2018). What do we know about manufacturing reshoring? Journal of Global Operations and Strategic Sourcing, 11(1), 79-122.

Chong, S., Pan, G.-T., Chin, J., Show, P.L., Yang, T.C.K., \& Huang, C.-M. (2018). Integration of 3D printing and industry 4.0 into engineering teaching. Sustainability (Switzerland), 10(11). 
Chou, P.-N., \& Feng, S.-T. (2019). Using a tablet computer application to advance high school students' laboratory learning experiences: A focus on electrical engineering education.

Sustainability (Switzerland), 11(2).

Diez-Olivan, A., Del Ser, J., Galar, D., \& Sierra, B. (2019). Data fusion and machine learning for industrial prognosis: Trends and perspectives towards Industry 4.0. Information Fusion, 50, 92111.

ElMaraghy, H., Moussa, M., ElMaraghy, W., \& Abbas, M. (2017). Integrated Product / System Design and Planning for New Product Family in a Changeable Learning Factory. Procedia Manufacturing, 9, 65-72.

Ghani, E.K., \& Muhammad, K. (2019). Industry 4.0: Employers' expectations of accounting graduates and its implications on teaching and learning practices. International Journal of Education and Practice, 7(1), 19-29.

Gitelman, L.D., \& Kozhevnikov, M.V. (2018). Paradigm of Managerial Education for a Technological Breakthrough in the Economy. Economy of Region, 14(2), 433-449.

Gorecky, D., Khamis, M., \& Mura, K. (2017). Introduction and establishment of virtual training in the factory of the future. International Journal of Computer Integrated Manufacturing, 30(1), 182-190.

Guo, Q., Miyamae, Y., Wang, Z., Taniuchi, K., Yang, H., \& Liu, Y. (2018). Senvis-Net: Learning from imbalanced machinery data by transferring visual element detectors. International Journal of Machine Learning and Computing, 8(5), 416-422.

Hariharasudan, A., \& Kot, S. (2018). A scoping review on Digital English and Education 4.0 for Industry 4.0. Social Sciences, 7(11).

Hold, P., Erol, S., Reisinger, G., \& Sihn, W. (2017). Planning and Evaluation of Digital Assistance Systems. Procedia Manufacturing, 9, 143-150.

Kaasinen, E., Schmalfuß, F., Özturk, C., Aromaa, S., Boubekeur, M., Heilala, J., Heikkilä, P., Kuula, T., Liinasuo, M., Mach, S., Mehta, R., Petäjä, E., \& Walter, T. (2019). Empowering and engaging industrial workers with Operator 4.0 solutions. Computers and Industrial Engineering.

Kamp, B., Ochoa, A., \& Diaz, J. (2017). Smart servitization within the context of industrial usersupplier relationships: contingencies according to a machine tool manufacturer. International Journal on Interactive Design and Manufacturing, 11 (3), 651-663.

Karre, H., Hammer, M., Kleindienst, M., \& Ramsauer, C. (2017). Transition towards an Industry 4.0: State of the LeanLab at Graz University of Technology. Procedia Manufacturing, 9, 206213. 
Kinkel, S., Schemmann, B., \& Lichtner, R. (2017). Critical Competencies for the Innovativeness of Value Creation Champions: Identifying Challenges and Work-integrated Solutions. Procedia Manufacturing, 9, 323-330.

Küsters, D., Praß, N., \& Gloy, Y.-S. (2017). Textile Learning Factory 4.0 - Preparing Germany's Textile Industry for the Digital Future. Procedia Manufacturing, 9, 214-221.

Lenz, J., Wuest, T., \& Westkämper, E. (2018). Holistic approach to machine tool data analytics. Journal of Manufacturing Systems, 48, 180-191.

Longo, F., Nicoletti, L., \& Padovano, A. (2019). Emergency preparedness in industrial plants: A forward-looking solution based on industry 4.0 enabling technologies. Computers in Industry, $105,99-122$.

Lu, Y., Xie, R., \& Liang, S.Y. (2019). Bearing fault diagnosis with nonlinear adaptive dictionary learning. International Journal of Advanced Manufacturing Technology.

Madsen, O., \& Møller, C. (2017). The AAU Smart Production Laboratory for Teaching and Research in Emerging Digital Manufacturing Technologies. Procedia Manufacturing, 9, 106112.

Morozov, D., Lezoche, M., \& Panetto, H. (2018). Multi-paradigm modelling of Cyber-Physical Systems. IFAC-PapersOnLine, 51(11), 1385-1390.

Nagy, J., Oláh, J., Erdei, E., Máte, D., Popp, J. (2018). The Role and Impact of Industry 4.0 and the Internet of Things on the Business Strategy of the Value Chain - The Case of Hungary. Sustainability, 10, 3491.

Prodanov, C. C., \& Freitas, E. C. D. (2013). Metodologia do trabalho científico: Métodos e Técnicas da Pesquisa e do Trabalho Acadêmico. $2^{\mathrm{a}}$. ed. Novo Hamburgo: Universiade Freevale.

Reuter, M., Oberc, H., Wannöffel, M., Kreimeier, D., Klippert, J., Pawlicki, P., \& Kuhlenkötter, B. (2017). Learning Factories' Trainings as an Enabler of Proactive Workers' Participation Regarding Industrie 4.0. Procedia Manufacturing, 9, 354-360

Ricalde, F. J. G., Ortiz, A. Y. V., Sánchez, J. L. Z., \& Bustos, M. de los A. H. (2015). Social appropriation of knowledge in four degrees of business development in Mexican production organizations in the Southern Mexico / Belize border. Analysis of the Model OECD [Apropriación social del conocimiento en cuatro grados de desarollo empresarial en organizaciones productivas mexicanas en la frontera Sur Mexico / Belice. Análisis del modelo OCDE]. Universitas Humanística, 79, 325-346.

Rodrigues, A. R., Tavar, C., Nogueira, G. M., Librelotto, R. F. (2016). A bibliometria como ferramenta de análise da produção intelectual: uma análise dos hot topics sobre sustentabilidade. Biblionline, 12(3), 34-47. 
Rodrigues, L. F., Jesus, R. A. de, \& Schützer, K. (2016). Industrie 4.0 - A Literature Review [Industrie 4.0 - Uma Revisão de Literatura]. Revista de Ciência \& Tecnologia, 19(38), 33-45.

Sá Carvalho, E.S. de, \& Filho, N.F.D. (2018). Proposal for a mobile learning system focusing on the characteristics and applications practical of industry 4.0. [Proposta de um sistema de aprendizagem móvel com foco nas características e aplicações práticas da indústria 4.0]. RISTI Revista Ibérica de Sistemas e Tecnologias de Informação, 27, 36-51.

Shamim, S., Cang, S., Yu, H., \& Li, Y. (2017). Examining the feasibilities of Industry 4.0 for the hospitality sector with the lens of management practice. Energies, 10 (4).

Siddoo, V., Sawattawee, J., Janchai, W., \& Thinnukool, O. (2019). An exploratory study of digital workforce competency in Thailand. Heliyon, 5(5).

Silva, V. L. da, Kovaleski, J. L., \& Pagani, R. N. (2018). Technology Transfer and Human Capital in the Industrial 4.0 Scenario: A Theoretical Study. Future Studies Research Journal, 11(1), 102-122.

Stachová, K., Papula, J., Stacho, Z., \& Kohnová, L. (2019). External partnerships in employee education and development as the key to facing industry 4.0 challenges. Sustainability (Switzerland), 11(2).

Stocker, A., Brandl, P., Michalczuk, R., \& Rosenberger, M. (2014). Human-centred ICT tools for smart factories [Mensch-zentrierte IKT-Lösungen in einer Smart Factory]. Elektrotechnik und Informationstechnik, 131(7), 207-211.

Synnes, E.L., \& Welo, T. (2016). Bridging the Gap between High and Low-volume Production through Enhancement of Integrative Capabilities. Procedia Manufacturing, 5, 26-40.

Szalavetz, A. (2019). Industry 4.0 and capability development in manufacturing subsidiaries. Technological Forecasting and Social Change, 145, 384-395.

Tavcar, J., \& Horvath, I. (2019). A review of the principles of designing smart cyber-physical systems for run-time adaptation: Learned lessons and open issues. IEEE Transactions on Systems, Man, and Cybernetics: Systems, 49(1), 145-158.

Thalmann, S., Pammer-Schindler, V., Fessl, A., \& Weghofer, F. (2017). Learning 4.0:

Addressing challenges for employees successfully [Herausforderungen für menschen in der industrie 4.0 erfolgreich meistern]. Productivity Management, 22(2), 62-64.

Uhlemann, T.H.-J., Schock, C., Lehmann, C., Freiberger, S., \& Steinhilper, R. (2017). The Digital Twin: Demonstrating the Potential of Real Time Data Acquisition in Production Systems. Procedia Manufacturing, 9, 113-120.

Vila, C., Ugarte, D., Ríos, J., \& Abellán, J.V. (2017). Project-based collaborative engineering learning to develop Industry 4.0 skills within a PLM framework. Procedia Manufacturing, 13, 1269-1276. 
Yakovle, V.V., Khasanov, M.M., Sitnikov, A.N., Prokofiev, D.O., Pustovskikh, A.A., Margarit, A.S., Simonov, M.V., \& Perets, D.S. (2017). The direction of cognitive technologies development in the Upstream Division of Gazprom Neft Company. Neftyanoe Khozyaystvo - Oil Industry, 12, 6-9.

Zeidler, F., Bayhan, H., Venkatapathy, A.K.R., \& Hompel, M.T. (2017). Reference field for research and development of novel hybrid forms of human machine interaction in logistics [Referenzfeld zur erforschung und entwicklung neua rtiger hybrider formen der zusammenarbeit von menschen und maschinen in der logistik]. Logistics Journal. 